\title{
Rituels de deuil et symbolisme alimentaire en Tunisie
}

\author{
Mourning Rituals and Food Symbolism \\ in Tunisia
}

Sonia Mlayah Hamzaoui

Résumé : Cet article porte sur l'analyse des pratiques alimentaires rituelles au cours des différentes phases du rite funéraire tunisien. Elle est basée sur des enquêtes directes auprès de personnes ressources de différentes régions de Tunisie ainsi que sur l'observation participante à des deuils familiaux et de proches. L'objectif de cette étude est de faire ressortir le caractère exceptionnel de ces pratiques alimentaires par rapport au quotidien tunisien, de saisir leur sens et la symbolique qu'elles sous-tendent et d'apprécier l'ampleur des changements qu'elles ont subis. Les enquêtes de terrain nous ont permis de classer ces pratiques alimentaires rituelles en fonction des objectifs que la communauté cherche à atteindre à travers leur observation.

Mots clés : pratiques alimentaires, rites alimentaires, rites funéraires, rituels de deuil, symboles, symbolique alimentaire, Tunisie

Abstract: This study focuses on the analysis of eating practices rituals during the different phases of the Tunisian funeral rite. It is based on direct surveys, collected from resource people from different regions of Tunisia as well as on participant observation established on mourning families and relatives. The objective of this study is to highlight the diverse nature of these eating practices compared to Tunisian everyday life, to understand their meaning and the symbolism that they underlie and to appreciate the extent of the changes they have undergone. Field surveys have allowed us to analyse these eating practices rituals according to the objectives that the community seeks to achieve through their observation. 
Keywords: food practices, food rites, food symbolism, funeral rites, mourning rituals, symbols, Tunisia

L'analyse des pratiques alimentaires traditionnelles de la communauté musulmane tunisienne au cours des rites funéraires est pertinente. Elle permet de mettre en lumière les objectifs de la communauté, de saisir la symbolique de ces pratiques et de vérifier leur caractère exceptionnel par rapport au modèle alimentaire du quotidien. Dans une société où les céréales, les produits céréaliers et l'huile d'olive trônent sur la scène culinaire, il est intéressant de distinguer la place qu'occupent ces ingrédients et le rôle qu'ils jouent en période de deuil. Conformément à la composition alimentaire de la diète méditerranéenne, l'apport quotidien de viande demeure faible dans le modèle alimentaire traditionnel tunisien et prend une dimension pléthorique au cours des repas rituels.

Il s'agit donc ici de relever les différences entre pratiques alimentaires et culinaires quotidiennes et celles relatives au deuil, de vérifier si les rituels alimentaires du deuil se distinguent de ceux pratiqués au cours des rites festifs et d'en déceler, le cas échéant, les dissonances. Enfin, il est question dans cet article de vérifier si les changements affectant les pratiques alimentaires actuelles ont eu raison des changements relatifs aux rites funéraires ou si ces derniers restent immuables. Il est à noter que la notion de pratiques alimentaires et culinaires englobe, outre la réalisation des mets et la manière de les consommer, les manières d'utiliser un produit alimentaire et un ustensile de cuisine.

La méthodologie adoptée dans notre étude est celle des enquêtes directes, basées sur l'observation participante au cours de deuils familiaux et de proches, à Sousse et à Tunis, ainsi que la réalisation d'entretiens semi-directifs avec des personnes ressources des villes de Siliana, Makthar, Kesra, Tunis, Sousse, Sfax et Mahdia.

\section{Rites funéraires, rituels alimentaires}

Les rites funéraires de la communauté musulmane tunisienne traditionnelle peuvent être classés en quatre grandes phases, chacune d’elles se caractérisant par des particularités rituelles alimentaires. Afin de saisir le sens de ces spécificités et d'analyser leur symbolique, il est primordial de comprendre le rituel funéraire qui leur est étroitement associé.

La première phase concerne les personnes en fin de vie dont la mort est attendue, voire souhaitée, dans certains cas de vieillissement, de maladie ou de déchéance extrêmes. La seconde constitue un temps intermédiaire et se prolonge avec la survenue de la mort, jusqu'à la sortie de la dépouille de la maison vers sa dernière demeure. La troisième phase sétend de l'enterrement jusqu'au 
premier jour de la levée du deuil, la date variant selon les régions. La quatrième et dernière phase continue jusquà la clôture de l'année et la levée définitive et totale du deuil. Cette levée se fait progressivement, au fil d'un certain nombre de cérémonies. Si leur nombre est variable d'une région à l'autre, létape la plus communément célébrée est celle du quarantième jour.

Plusieurs pratiques rituelles alimentaires sont observées au cours de ces quatre phases du rite funéraire. Les objectifs recherchés à travers les pratiques alimentaires rituelles dépendent largement de la phase du deuil et de la ou des personnes qu'elles visent. Afin de mieux les appréhender, nous allons les analyser à travers leurs objectifs majeurs.

\section{Purifier le corps du défunt et attirer la paix sur son âme}

La première grande phase du deuil, qui en fait le précède, n’est pas générale et se limite aux personnes agonisantes. Il s'agit de préparer cette phase de passage, d'un état de vie à un état de mort, de la faciliter et d'en adoucir la transition. Un certain nombre de pratiques alimentaires est alors exécuté dans ce sens, la plus commune demeurant celle de donner à boire au mourant par petites gorgées. Cette opération est appelée dans certaines régions de Tunisie taqtir (littéralement, verser goutte à goutte), elle est assurée à l'aide d'une petite cuillère ou d'un morceau de coton propre et imbibé d'eau. Dans d'autres régions, et pour la même opération, on parle plutôt de tgharghir (littéralement gargariser). La référence est alors faite au bruit produit par le mourant qui, la plupart du temps, ne parvient pas à avaler l'eau correctement. Cette dernière est versée délicatement dans la bouche, à l'aide d'une théière ou d'une petite cuillère. Contrairement aux autres régions de Tunisie, à Tunis, la plupart des familles d'origine tunisoise s'interdisent la distillation domestique taqtir des eaux de roses, de fleurs d'orangers, de géranium ou autres, et achètent ces eaux dans le commerce. Dans la perception communautaire, elles chassent la mort de la maison par l'interdiction de cette pratique de distillation qui rappelle le goutte à goutte effectué pour la personne agonisante.

D'autres familles donnent également à la personne agonisante quelques gouttes de miel. L'eau et le miel sont considérés par la communauté musulmane tunisienne comme un don divin et des ingrédients sacrés. Ne sont-ils pas cités dans le Livre Saint ? Symbole de paix, de sécurité et de purification, l'eau au cours de ce rituel apaise le cour du mourant et purifie son âme. Le miel, symbole de douceur, permet d'adoucir le passage vers l'au-delà.

La deuxième grande phase du deuil constitue un état de transition où la personne, dont lâme vient juste de quitter le corps, n'a pas encore acquis le statut de mort et ne compte pas non plus parmi les vivants. Cet état d'entre deux est considéré comme une étape dangereuse, de vulnérabilité pour le mort. Dans les perceptions communautaires, le risque consiste dans la possibilité que le diable puisse s'accaparer de lâme qui vient de quitter le corps ou 
de corps qui ne possède plus d'âme. Il existe, dans les deux cas, des pratiques rituelles à respecter pour contourner cet imminent danger. Dans cette perspective, on place, dans certaines régions de Tunisie, à proximité de la tête du mort, un morceau de pain ou un ustensile contenant de l'eau. Don divin par excellence, le pain est un ingrédient sacralisé, doté d'une forte charge symbolique et dichotomique de protection/nuisance permettant de constituer un bouclier protecteur du corps, et en même temps de s'en prendre au diable dès qu'il s'en approche. Grâce à son pouvoir magico-religieux, ce même pain ne permet-il pas de sceller l'amitié entre ceux qui le partagent, et de nuire à celui qui trahit ce pacte d'amitié ? (Mlayah Hamzaoui $2006: 205$ ).

Leau est, elle aussi, dotée d'une vertu de protection des humains au cours des différents âges de la vie. La mère dépose à proximité de la tête de son nourrisson un ustensile rempli d'eau avant de le laisser seul dans sa chambre et de vaquer à ses activités quotidiennes. Dans les perceptions communautaires, telles qu'elles ressortent de nos enquêtes, l'idée est d'éloigner le diable et les mauvais esprits de ce petit ange complètement démuni de toutes défenses. Le même principe est appliqué pour le voyageur, à la suite duquel on jette de l'eau lorsqu'il quitte la maison. Cette eau l'accompagne durant son voyage, le protège et lui permet de rentrer chez lui sain et sauf ; c'est celle-là même qui l'enverra en paix dans l'au-delà.

\section{Attirer, pour le défunt, la miséricorde et le pardon de Dieu}

Afin que le Divin accorde sa miséricorde au défunt, la famille proche multiplie les prières et les offrandes alimentaires tout au long des différentes phases du deuil. Les pratiques alimentaires rituelles varient sensiblement d'une région à une autre, mais leur principe reste le même. Il s'agit pour cette communauté musulmane d’alléger les péchés du mort et de l'aider à bénéficier de la grâce divine afin de lui garantir le paradis. Certaines familles vont jusquà prolonger ces offrandes au-delà de l'année. S’agissant d'une aumône courante (sadaqa jeriya), plus elle dure dans le temps, plus le nombre de personnes qui l'accomplissent est important, et plus elle portera ses fruits.

Dans certaines régions de Tunisie, dès la survenue de la mort, la famille proche procède au sacrifice d'un mouton, parmi ceux que le défunt possédait ou qu'on achètera avec l'argent qu'il a laissé . Selon les moyens de chacun, ce sacrifice peut être renouvelé ou non, à la première cérémonie de la levée du deuil, au quarantième jour et à la clôture de l'année. S'il n’est pas renouvelé au cours de l'un de ces jours ponctuant la levée du deuil, il peut être pratiqué au cours de la première fête religieuse du sacrifice Aïd el kébir, qui arrive après la mort. La famille du défunt pourra alors égorger un deuxième mouton au nom du défunt et l'offrir entièrement en aumône. Les règles régissant ce sacrifice socialement recommandé sont les mêmes que pour celui religieusement pratiqué lors de la fête du sacrifice ${ }^{3}$. Par ailleurs, contrairement aux pratiques 
culinaires festives de l'Aïd, les pratiques relatives au deuil n'autorisent que l'utilisation de la viande de l'animal sacrifié. L'estomac, les intestins, les abats, les rognons, la tête et les pattes habituellement cuisinés et accommodés en plats préparés pour satisfaire les envies des uns et des autres seront offerts en aumône aux pauvres, qui prieront pour lâme du mort.

Pour d’autres régions de Tunisie, le sacrifice ne constitue pas une obligation en soi, mais l'achat de la viande en est une. Dans ce cas, ce nest pas tant le sacrifice qui compte pour la famille endeuillée que la viande nécessaire à la préparation du plat rituel du deuil. Dans le cas où il s'agit d'une personne de condition modeste, la solidarité communautaire s’opérera de façon spontanée, les fidèles cherchant à profiter du bénéfice de leurs bonnes actions auprès de Dieu et sollicitant la miséricorde divine pour le défunt. Par ailleurs, les parts de viande provenant du sacrifice ou achetées doivent être généreuses et de bonne taille. Elles seront distribuées de façon égale, sans distinction de statut social, contrairement aux consommations du quotidien et relatives aux rites festifs où le genre et le rang social sont déterminants. Cette pratique trouve sa justification dans le principe religieux qui précise que tous les êtres humains sont égaux devant Dieu.

Destiné à la préparation exclusive du plat d'un couscous qualifié, partout en Tunisie, de « dîner du mort », le délai d’acquisition de la viande est régi par la règle sociale qui stipule que le cadavre ne peut quitter la maison que si de la viande a été achetée : " une viande ne sort que si une autre entre ${ }^{4}$ ».

Dans le modèle alimentaire traditionnel tunisien, la viande nétait pas consommée quotidiennement. Sa présence dans les préparations culinaires n'est souvent attestée qu'une à deux fois par semaine et en quantité limitée, alors que pour le deuil elle devient l'aliment de l'abondance, de la bombance, accompagnant le plat de couscous rituel. Ce dernier est généralement préparé en quantité au cours de la première cérémonie de la levée du deuil, sauf dans les régions où l'on procède au sacrifice dès l'annonce de la mort. Ici, on prépare le couscous, également en grande quantité, le jour du décès. L'idée est de nourrir les personnes venues de loin pour présenter leurs condoléances, ainsi que les récitants du Coran. En réalité, toute l'assistance partage ce couscous dont une partie sera donnée en aumône aux nécessiteux. Tous prieront pour le repos de l'âme du mort.

Repas communiel par excellence, chaque graine de couscous consommée constitue un gain de plus dans la miséricorde divine exercée au profit du défunt. Ainsi, on incite toutes les personnes présentes qui, pour une raison ou une autre, refusent de manger, à le faire en joignant la formule : «Mangez pour sa miséricorde ». D'un autre côté, un grand ustensile contenant ce mets et garni de morceaux de viande est souvent donné en offrande à partager entre les nécessiteux fréquentant la même mosquée. Chaque personne ayant consommé un peu de ce couscous sollicitera Dieu à voix audible, afin qu'il accorde sa miséricorde et sa bénédiction au défunt grâce à la prononciation de la formule conventionnelle « rabi yarahmou ou innaamou ${ }^{5} »$. 
Des cuillères sont préalablement plantées dans ce grand plat de couscous déposé sur une table, dans la cuisine ou la salle à manger, en tout cas loin des regards. Les gens passeront à tour de rôle et par petits groupes pour se servir. La plupart des convives, debout et en silence, prélèveront quelques bouchées du couscous et mangeront à la va vite un morceau de viande. En effet, il s'agit, ici, plus de l'accomplissement d'un « devoir » que d'un moment de détente et de sociabilité.

Les activités culinaires relatives à la réalisation du couscous et d'autres préparations sont assurées par des femmes habiles à préparer de la nourriture en grandes quantités. Obligatoirement, elles ne font pas partie des parents très proches du défunt. Ces derniers, accablés par le chagrin, ne sont pas en mesure de faire quoi que ce soit. Le plat de couscous du rituel du deuil ne diffère pas dans sa préparation de ceux du quotidien et des rituels festifs. Cependant, il ne doit contenir ni raisins secs, ni graines de grenade, ni toute autre douceur synonyme de joie.

Toujours dans un souci d'attirer la miséricorde de Dieu sur le mort, en dehors du couscous rituel, certaines familles chargeront un de leurs proches de distribuer du pain imbibé d'huile d'olive. Cette offrande destinée aux mendiants qui se trouvent dans le cimetière doit se faire à partir du jour de l'enterrement, entre la prière du crépuscule et celle du soir, et durant au moins trois jours. Les personnes qui bénéficieront de ce don d'ingrédients sacrés n’omettront pas de prier pour l'âme du défunt. Dans d'autres régions de Tunisie, les femmes, lors de leur visite du tombeau, au lendemain de lenterrement, noublieront pas de se munir d'un couffin quelles auront rempli de pain, de beignets, de morceaux de sucre et de confiseries. A travers ce don de douceurs, elles cherchent à obtenir la clémence divine pour le défunt, à alléger ses éventuelles souffrances ${ }^{6}$ et à garantir la paix de son âme.

Certaines familles continueront d’offrir le dîner du mort jusquà la clôture de l'année et même plus encore, et ce afin d’attirer la miséricorde pour son âme. Ils prélèveront alors cette offrande sur leur propre dîner : il s'agit de la part du mort, servie comme lors de son vivant.

\section{Manifester sa peine, lever le deuil et se protéger de la survenue d'une deuxième mort}

La troisième grande phase de deuil débute dès l'enterrement de la dépouille, moment à partir duquel le défunt acquiert finalement le statut de mort. Cette phase se prolonge jusquà la célébration de la première étape de levée du deuil. Plusieurs rituels alimentaires destinés à la famille proche sont alors accomplis, afin de prémunir celle-ci du danger d'un deuxième décès et de lui permettre de manifester sa peine. Une série de pratiques alimentaires est alors observée afin qu'un autre malheur ne s'abatte à nouveau sur la famille. A cette fin, au Nord-Ouest de la Tunisie, on prépare une bouillie à base de farine ou 
de semoule, accompagnée d'huile d’olive et de sucre et destinée aux personnes qui ont creusé la tombe du mort. Appelée assidet tartih el fissène, littéralement «bouillie pour le repos des pioches », il s'agit d'une récompense donnée aux pioches et aux pelles, afin qu'elles ne reprennent pas leur activité de sitôt (Mlayah Hamzaoui 2009 : 110). Dans le modèle alimentaire traditionnel tunisien, cette préparation est habituellement consommée au cours du petit déjeuner, ainsi qu'à loccasion de la commémoration de la naissance du Prophète, qui l'affectionnait particulièrement.

Dans certaines régions de Tunisie, on sert du pain et de l'huile d'olive aux femmes présentes dès la sortie du cercueil de la maison, et aux hommes à leur retour du cortège funéraire. L'idée est de récompenser les uns et les autres pour le partage de la peine, pour l'effort fourni et la compassion manifestée, éliminant ainsi toutes raisons de retour, et par là même de renouvellement, du deuil. Dans d’autres régions, ce rituel est également pratiqué quand le défunt était centenaire, afin que ceux qui consomment ces deux produits alimentaires puissent vivre aussi longtemps que lui.

$\mathrm{Si}$, au cours des premiers jours qui succèdent au décès, la douleur des membres de la famille endeuillée est incommensurable, elle va en diminuant avec le temps. La manifestation de la peine n'est pas seulement extérieure : c'est tout le système culinaire et alimentaire qui s'en trouve bouleversé. Ce bouleversement est marqué d'interdits alimentaires éliminés progressivement, parallèlement aux différentes cérémonies de levée du deuil. Ils concernent la cuisson des aliments, leur saveur et leur couleur, ainsi que la composition des plats et des menus. Par ailleurs, il est intéressant de noter l'importance des aliments dont létymologie du mot renvoie à la peine. La régression du chagrin et le fléchissement des interdits vont en diminuant à chacune des cérémonies de levée du deuil. Ils s’arrêtent de manière définitive à la clôture de l'année.

Le premier interdit alimentaire porte sur la cuisson des aliments dans la famille du défunt, où l'allumage du feu sera complètement aboli. Traditionnellement, dès les dernières heures d'agonie de la personne mourante et jusqu'à la première cérémonie de levée du deuil, les membres de sa famille proche cessent toute activité culinaire. A Mahdia, cette cérémonie a lieu au septième jour après l'inhumation du corps, c'est pourquoi elle y est appelée nhar essebaa. A Sfax, elle est célébrée dès le premier jeudi suivant l’enterrement, d'où son appellation khmis, jeudi. Dans toutes les autres régions de Tunisie, elle est célébrée au troisième jour après l’enterrement. Appelée nhar el fark, jour " de la séparation », elle symbolise la séparation d’avec le chagrin. Au cours des jours précédant cette cérémonie, la famille proche manifeste sa peine par l'abandon-par interdiction sociale-des activités culinaires. De ce fait, et exception faite pour les régions qui procèdent au sacrifice dès la survenue de la mort, la plupart des autres régions s'abstiennent d'allumer le feu dans la maison du défunt. Il est toutefois important de souligner que dans les régions pratiquant le sacrifice, ni l'allumage du feu, ni la cuisine ne sont effectués par la famille proche. C'est la communauté qui se charge de ces activités culinaires 
et du sacrifice, autant que possible, loin de la maison du mort, tout au moins loin des regards de ses proches. Il est un autre objectif derrière la négation du feu qui est celui de l'atténuation du chagrin. Ainsi, dans les perceptions communautaires, l'allumage du feu peut, par un effet de contamination, raviver ou augmenter le feu de la douleur de la séparation.

A la reprise des activités de cuisson, on suspendra la confection des mets qui nécessitent une préparation élaborée. Seul le plat de couscous et, avant lui, pour la région de Mahdia, celui de mhames, sont cuisinés par la communauté. Les céréales connues sous l'appellation française de "graines de plomb " sont, en arabe, appelées mhames, de même que le plat préparé avec cet ingrédient. La racine étymologique de mhames renvoie à la peine (hamsa), et une petite poignée de ces graines de céréales est avalée crue $^{7}$ dans certaines régions de Tunisie, simulant ainsi l'acte de ravaler sa peine, de mettre un terme au chagrin, du moins d’en atténuer l'ampleur. En partant du même principe, à Mahdia, on offre un plat de mhames aux femmes une fois la sortie du cercueil terminée, et aux hommes dès leur retour du cimetière. Le plat de couscous préparé au cours des cérémonies de levée du deuil comporte dans sa composition des pois chiches, en arabe homs, dont la racine étymologique est également la peine, hamsa. A contrario, ces légumes séchés doivent être absents du plat de couscous préparé pour les rituels festifs. Par un effet de contamination, leur présence pourrait être de mauvais augure et altérer la joie de la fête. Au quotidien, le couscous ne comporte aucune restriction d'ingrédients : seuls les goûts des uns et des autres détermineront ses éléments.

En ce qui concerne les interdits relatifs à la saveur et la couleur des plats, sont interdits les bonnes saveurs et le goût sucré, d'une part, et la couleur verte, d'autre part. Ainsi, et pour revenir au mhames spécifique aux habitants de Mahdia, ce plat doit être préparé blanc azaar, sans épice ni tomate ${ }^{8}$, et avec de la viande séchée (qaddid), des merguez fraiches et des œufs à la coque. Cette composition reste différente de celle du quotidien, où ce plat est préparé avec des épices spécifiques, dont le piment et la tomate concentrée, réputés donner couleur et saveur aux plats. Au cours du deuil, l'interdiction d'intégrer ces ingrédients dans la préparation rituelle garantit l'obtention évidente d'un plat fade et sans couleur. Le manque de saveur est sans doute recherché afin de ne pas ouvrir les appétits, et de ne pas avoir à le préparer une nouvelle fois, et donc de ne pas présager un nouveau décès. Par ailleurs, la présence, dans ce plat rituel, d'une viande séchée et non fraîche n’est pas sans renvoyer à l'état sec du mort, et celui humide du vivant. Ce plat constitue le dernier point de rencontre entre le mort et les vivants, pour qui la vie continue à travers la présence de l'œuf, symbole de vie. Pour le reste, et contrairement au couscous préparé pour les rites festifs et de joie, les légumes sont admis dans le rituel du deuil. En effet, ces produits alimentaires sont connus pour affadir la préparation. Or, au cours des rites funéraires, plus le couscous est fade et sans saveur, moins le risque est grand qu'un deuxième décès ne survienne dans la famille. 
Dans les perceptions communautaires tunisiennes, le goût du sucré et les douceurs renvoient à la joie et à la gaieté. De ce fait, ces douceurs étaient traditionnellement interdites de toutes les pratiques alimentaires rituelles du deuil, sauf pour les citadins de Tunis et ceux de Mahdia. Pour les premiers, la première apparition du sucré se concrétise dans le verre de thé et la datte, offerts au cours de la première cérémonie de levée du deuil, nhar el fark. Elle se poursuit le jour de la ziara (littéralement, visite), célébrée au quinzième jour après l'inhumation du corps et qui correspond pour les citadins de Tunis à la deuxième cérémonie de levée du deuil ${ }^{9}$. En ce jour, les Tunisois offrent aux personnes présentes un verre de thé et un gâteau appelé briket hlib ${ }^{10}$ (Mlayah Hamzaoui 2006 : 119) dont la préparation nécessite l'utilisation d'un rouleau à pâtisserie fin et allongé, en bois. Leur idée est quà travers la manipulation du rouleau, on arrive à tourner la page du deuil, de la même manière qu'on retourne la couche fine d'une pâte pâtissière, ndaourou el oud ${ }^{11}$. Au cours de la troisième cérémonie de levée du deuil appelée arbiine, " quarante ", les Tunisois offrent à leurs invités ${ }^{12}$ un plat de mloukhia et un ragoût sucré/salé appelé marqa hloua ${ }^{13}$. La mloukhia est un ragoût de couleur verte, réalisé avec de la poudre de feuilles de corète séchées. Considérée comme un plat béni, en raison de sa couleur qui renvoie, dans la tradition musulmane, à celles du paradis, cette préparation est présente dans tous les rites festifs et inauguraux. A travers son introduction au cours de cette cérémonie, la famille proche marque son entrée dans un nouveau cycle de vie où il n'y a plus place pour le deuil.

Au cours de la première cérémonie de levée du deuil, l'introduction du goût sucré-qui choquait jadis les autres régions de Tunisie-est actuellement adoptée par la plupart des Tunisiens. Seuls les plus conservateurs n'ont admis aucune modification dans leurs rites funéraires. Ce refus du changement semble ainsi les prémunir d'une modernisation ravageuse, érosive des pratiques sociales traditionnelles. Toutefois, l'interdiction de la confection de gâteaux traditionnels à loccasion du premier Aïd el fitr ${ }^{14}$, et durant une année après le décès, est encore observée par la plupart des familles endeuillées.

Au cours de la cérémonie du quarantième jour à Mahdia, la famille du défunt invite les proches à partager un plat de couscous s'il s'agit d'un dîner, ou un bol de masfouf lors d'un repas de veillée. Le masfouf est une préparation sucrée, réalisée à partir de graines de couscous fines cuites à la vapeur, assaisonnée avec un peu de beurre rance et agrémentée de fruits secs et de raisins. Elle est essentiellement consommée au cours du mois de Ramadan, en cours de soirée ou à la dernière prise alimentaire du soir. Ici, cette préparation de couleur blanche renvoie à la blancheur du plat de mhames consommé par les familles endeuillées le jour de l'enterrement. Si, à travers la couleur blanche du mhames, le manque de saveur et la fadeur sont visés, dans le masfouf sont recherchés la purification des vivants du deuil du mort, le renouveau et la prospérité. Dès lors, la présence du sucré et des fruits secs est là pour confirmer cette quête. Dans son analyse du repas funéraire des Mongols, Sandrine Ruhlmann avance 
également cette idée qu’au cours du rituel funéraire, les nourritures qualifiées de blanches relèvent de rites de purification et symbolisent le renouveau et la prospérité (2008:262). Lauteure estime que cette purification concerne « les membres du cortège funéraire, les membres de la famille restreinte et étendue, et les convives, parce que sur leur personne repose un soupçon de contagion par le contact ou la proximité avec la dépouille " (2008: 262-263). Ceci est peu vérifiable pour la communauté musulmane tunisienne, puisque ce sont les vivants qui constituent une source d'impureté pour le cadavre du défunt, et non l'inverse (Fortier 2006 : 30). Ainsi, la purification recherchée par les familles endeuillées de Mahdia vise bien à éliminer les impuretés du deuil, et non celles de la dépouille.

Dans le modèle traditionnel Tunisien, tous les plats préparés au cours du rituel du deuil devaient être de composition simple, faciles à réaliser et à consommer. Il en allait de même pour la composition du menu de la première cérémonie de levée du deuil, qui ne devait comprendre qu'un seul plat, celui du couscous rituel. S'il est vrai que le sacrifice, l'achat de la viande et la préparation du plat rituel du couscous sont jusquà aujourd'hui maintenus au cours des rites funéraires, la taille des morceaux de viande, elle, a sensiblement diminué, tandis qu'au seul et unique plat de couscous sont associés des salades, des tajines ${ }^{15}$ et un ou plusieurs desserts. Ce menu est présenté dans des assiettes individuelles, auprès de convives attablés, prenant le temps de déguster leurs repas et profitant du moment pour prendre des nouvelles des uns et des autres, jusqu'à en oublier l'essentiel : prier que Dieu accorde sa clémence au défunt. Pour les plus pieux, l'ostentation de ces pratiques traditionnellement modestes ne semble pas en adéquation avec les valeurs de discrétion et d'humilité que la mort devrait éveiller en chaque musulman. Plus encore, certaines familles vont jusquà engager des cuisinières ou un traiteur et des serveurs pour le repas du premier temps de levée du deuil.

\section{Accumuler les bonnes actions, renforcer et renouveler les liens sociaux}

La réalisation des bonnes actions, le renforcement et le renouvellement des liens sociaux sont effectués, au cours des différentes phases du deuil, par la communauté et par les professionnels de la mort qui ne cherchent pas à être rémunérés. Le sacrifice opéré à la survenue de la mort, et les activités culinaires qu'il engendre, ainsi que celles allant jusquà la première cérémonie de la levée du deuil, sont réalisés par la communauté. Du fait de la suppression du feu dans la maison du défunt, la communauté se charge d'offrir en guise de bonne action à la famille endeuillée, le petit déjeuner (café compris), le déjeuner, le dîner et tout ce dont celle-ci a besoin en nourritures et en boissons. 
Une pléthore d'aliments est ainsi mise à la disposition des personnes accablées par la douleur, et déclarant ne rien pouvoir avaler. Ces aliments sont partagés avec les personnes venues présenter leurs condoléances, en espérant que la commensalité créée autour de ces repas permette d'ouvrir l'appétit des uns et des autres.

À l'occasion de l'Aïd el fitr, certains membres de la communauté offriront des gâteaux traditionnels à la famille endeuillée pour qui leur confection est interdite, comme le veut la coutume. Il en est de même pour la fête du mouled qui commémore la naissance du Prophète. En effet, pour célébrer cet événement les Tunisiens procèdent à la réalisation de l'assida bidha bouillie de farine, ou assidet zgougou, bouillie réalisée à partir des graines de pin d'Alep, décorée d'une crème anglaise agrémentée de fruits secs. Etant donné qu'il est interdit à la famille endeuillée de préparer l'un ou l'autre mets, les membres proches de la communauté se chargent de les leur offrir.

Il est également interdit à la famille proche du défunt de procéder à l'approvisionnement annuel habituel, oula, surtout en ce qui concerne la transformation des céréales en produits céréaliers, tels le couscous, le mhames, le boulgour et autres. Dans ce cas, la communauté se charge de fournir la famille en ingrédients. Les ustensiles qui contiennent les aliments offerts doivent être rendus vides, pour ne pas les remplir avec la peine du deuil. Hormis le cas des rites funéraires, il est donc de mauvais augure de rendre un ustensile vide, cet acte pouvant entraîner la mort dans la famille à qui on l'a rendu (Mlayah Hamzaoui 2006 : 203). Toutes ces bonnes actions au profit de la famille endeuillée constitueront une dette à rendre à leurs auteurs dans un contexte équivalent.

D’autres pratiques rituelles alimentaires effectuées au cours du deuil seront considérées comme bénéfiques aux vivants : au moment de la lecture du Coran, on place devant ses récitants un ustensile contenant de l'eau et un autre renfermant des morceaux de sucre. Ces ingrédients seront appelés à l'issue de la lecture des soixante hizb $b^{16}$, smat, et seront alors considérés comme des aliments bénis et dotés de pouvoir magico-religieux. Ils seront par la suite offerts aux personnes présentes et à tout individu souffrant de maux de tête. La distribution de ces deux produits alimentaires permet à son auteur de cumuler les bonnes actions, qui l'aident dans sa quête du paradis.

Actuellement, si certaines des pratiques culinaires évoquées dans cet article restent inébranlables, d’autres se voient, elles, complètement métamorphosées : l'ascétisme social requis face à la mort a laissé la place à l'ostentation et à l'exhibition des richesses parmi les vivants. L'abstinence, la modestie et l'humilité dont les aïeux faisaient preuve au cours des rites funéraires sont aujourd'hui dénigrées, qualifiées de rétrogrades sous l'effet d'une modernisation galopante, altérant les grandes valeurs dites d’antan. 
Sonia Mlayah Hamzaoui est nutritionniste et docteure en sociologie de l'alimentation. Chargée de recherches à l'Institut national du patrimoine, elle a été affectée au Musée national du patrimoine de 2004 à 2008 et, depuis, à la Division du Développement muséographique, section ethnographique. Elle a été chargée de collections et de recherches au Musée des civilisations de l'Europe et de la Méditerranée à Marseille au cours de l'année 2014 et elle enseigne "l'anthropologie de l'art culinaire " à la Faculté des Sciences humaines et sociales de Tunis. Parmi ses réalisations : la conception du musée du patrimoine traditionnel de Kesra et de plusieurs expositions temporaires, la production d'un film documentaire ethnographique sur les rites autour du mariage à Kesra et plusieurs études sur le patrimoine immatériel tunisien. Elle est l'auteure de plusieurs articles sur l'alimentation et la diète méditerranéenne et d'un ouvrage intitulé "Modernité et Tradition : les pratiques culinaires des citadins de Tunis : essai d'anthropologie culinaire " (Centre des publications universitaires, Tunis 2006) primé en 2007 du premier prix des écrits sociologiques francophones de Zoubeida Bchir.

Courriel : soniamzaoui@yahoo.fr

Sonia Mlayah Hamzaoui is a nutritionist and doctor in sociology of food. Hamzaoui was in charge of research at the National Heritage Institute assigned to the National Heritage Museum from 2004 to 2008 and since then to the Division of Museographic Development, ethnographic section. She has been in charge of collections and research at the Museum of Civilizations of Europe and the Mediterranean in Marseille in 2014. Hamzaoui teaches "the anthropology of culinary art" at the Faculty of Human and Social Sciences in Tunis. Among her achievements are the design of the traditional heritage museum of Kesra and several temporary exhibitions, the production of an ethnographic documentary film on the rites around marriage in Kesra and several studies on the Tunisian intangible heritage. She is also the author of several articles about food and Mediterranean diet including a book entitled Modernité et Tradition: les pratiques culinaires des citadins de Tunis: essai d'anthropologie culinaire, published by the Centre des Publications Universitaires, Tunis in 2006 and awarded in 2007 with the first prize for French-speaking sociological writings by Zoubeida Bchir. Email: soniamzaoui@yahoo.fr 


\section{Notes}

1. Dans les perceptions communautaires, le pain nuit autant qu'il protège. Un proverbe tunisien met en garde contre cette nuisance "rod belek el naima etih fik" (fais attention, le pain peut te nuire).

2. Toutes les dépenses générées par les rites funéraires (et pas seulement les dépenses relatives à la préparation des plats, au sacrifice ou à l'achat de la viande) doivent provenir des biens laissés par le défunt.

3. Voir Mlayah Hamzaoui 2006 : 208-212.

4. L'expression proverbiale tunisienne est: "Ma yokhrej lham kan ma yodkhel lham ».

5. "Que Dieu lui donne sa miséricorde et qu'il le bénisse».

6. En Islam, si le défunt a cumulé des péchés au cours de sa vie, son châtiment peut commencer alors qu'il est encore dans sa tombe.

7. En dehors du rituel du deuil, ces céréales ne seront jamais consommées crues.

8. En dehors du deuil, ce plat est préparé avec de la tomate concentrée, du piment rouge et des épices.

9. Pour les autres régions de Tunisie, la deuxième cérémonie de la levée du deuil correspond au quarantième jour après l'inhumation chiffre auquel on soustrait le nombre de fils du défunt.

10. Il s'agit de cigares de pâte feuilletée frite, farcis d'une crème anglaise, trempés dans du sirop de sucre et saupoudrés de pistaches moulues.

11. Littéralement, « tourner le bâton ».

12. Les personnes présentes à cette cérémonie doivent y être invitées, sauf dans le Sud tunisien où tous ceux qui désirent y prendre part n'ont pas besoin d'invitation.

13. Littéralement, " ragoût sucré ". Il s'agit d'un ragoût préparé avec des châtaignes, des fruits secs et de la viande d'agneau.

14. Il s'agit de la fête religieuse qui succède au mois de Ramadan.

15. Le tajine tunisien diffère de celui des marocains. Il s'agit d'un mets qui possède la consistance et l'aspect de la tortilla espagnole.

16. Il s'agit d'une partie d'un juzz (chapitre du Coran) : chaque juzz est composée de deux hizb. Le Coran comporte au total soixante hizb.

\section{Références bibliographiques}

Fortier, C. (2006), 'La mort vivante ou le corps intercesseur (société maure-islam malékite)', Revue des Mondes Musulmans de la Méditerranée, no. 113-114 : 229-245.

Mlayah Hamzaoui, S. (2006), Modernité et Tradition : les pratiques culinaires des citadins de Tunis : essai d'anthropologie culinaire (Tunis : Centre des publications universitaires).

Mlayah Hamzaoui, S. (2009), 'Les fonctions symboliques de certains plats rituels de Makthar et Kesra', Africa, série : Arts et Traditions Populaires, no. XV : 103-112.

Ruhlmann S. (2008), 'Une soupe peu ordinaire, analyse du repas des funérailles chez les Mongols', Techniques et cultures, no. $51: 246-271$. 